\title{
Artificially Integrated Synthetic Rectangular Waveguide
}

\author{
Hsien-Shun Wu, Student Member IEEE, and Ching-Kuang C. Tzuang, Fellow, IEEE
}

\begin{abstract}
A synthetic rectangular waveguide (SRW), which consists of two electrical sidewalls and two parallel periodical structures placed at the top and bottom surfaces of the waveguide, is presented. The SRW is made by multilayered integrated circuit processes, which typically have large ratios of SRW lateral dimensions to substrate thickness. Two theoretical methods, finite-element method and deembedding of composite structure consisting of SRW and mode converters, are applied to investigate the propagation characteristics of the SRW. Application of the dispersion characteristics of the two-dimensional periodical structures coupled with appropriate mode converter designs leads to results in SRW designs supporting $\mathrm{TE}_{10}, \mathrm{TM}_{00}$, and $\mathrm{TM}_{10}$ modes. Measurements and the two theoretical approaches indicate that the slow-wave factor is 4.9 and $Q$-factor is 260 at $6.85 \mathrm{GHz}$ for the $\mathrm{TE}_{10}$ mode propagation with a cutoff frequency of 4.10 $\mathrm{GHz}(0.348$ factor of cutoff frequency of conventional rectangular waveguide using the same material and dimensions). The theoretical data show the $\mathrm{TM}_{00}$ mode to have a slow-wave factor of 1.8 , $Q$-factor of 187.6 at $11.4 \mathrm{GHz}$, and cutoff frequency of $10.2 \mathrm{GHz}$. The $\mathrm{TM}_{10}$ mode has a slow-wave factor of $1.98, Q$-factor of 187.6 at $12.5 \mathrm{GHz}$, and cutoff frequency of $10.4 \mathrm{GHz}$.
\end{abstract}

Index Terms-Microwave integrated circuit (MIC), periodical structure, rectangular waveguide, slow-wave device, $\mathbf{T E}_{10}, \mathbf{T M}_{00}$, $\mathbf{T M}_{10}$.

\section{INTRODUCTION}

$\mathbf{P}$ ERIODICAL structures, especially planar ones, have found many useful applications for high-performance radio-frequency (RF) front-end components and modules, including in antenna gain enhancement [1]-[4], novel leaky-wave antennas [5]-[8], beam steering array antennas [9], size-reduced patch antennas [10], [11], miniaturized bandpass filters with intrinsic spurious suppressions [12]-[14], electromagnetic bandgap (EBG) waveguide filters [15], [16], transverse electromagnetic (TEM) waveguides [17], [18], efficiency improvement of quasi-optical amplifiers using TEM waveguide [19], [20], metamaterial structures [21], [22], miniaturized rectangular waveguide [23], high-Q resonators [24]-[26], leakage suppressors [1], [12], [27]-[31], and others. A survey of works on the application of periodical structures reveals many more papers than mentioned above, reflecting growing interest in various aspects of this field such as frequency-selective surface [32], soft-hard surfaces [33]-[35], and many others.

Manuscript received October 30, 2004; revised February 22, 2005. This work was supported in part by the Ministry of Education of Taiwan under Grant 89-E-FA06-2-4 and in part by the National Science Council under Grant NSC 91-2219-E-009-025.

H.-S. Wu is with the Institute of Electrical Communication Engineering, National Chiao Tung University, Hsinchu, Taiwan, R.O.C.

C.-K. C. Tzuang is with the Graduate Institute of Communication Engineering, Department of Electrical Engineering, National Taiwan University, Taipei, Taiwan, R.O.C. (e-mail: cktzuang @cc.ee.ntu.edu.tw).

Digital Object Identifier 10.1109/TAP.2005.854523
This paper presents a new field of study, focusing on the development of novel or new guiding structures that must be realized by multilayered integrated circuit processes, which can be integrated into the microwave integrated circuit (MIC) without difficulties. Uchimura et al. reported a synthetic rectangular waveguide (SRW) made of laminated dielectric substrates with periodical shorting posts (vias) supporting the $\mathrm{TE}_{10}$ mode [36]. The propagation characteristics of such SRWs are similar to those of a conventional rectangular waveguide.

This paper proposes a new approach by which the SRW can break the theoretical limit on the conventional rectangular waveguide made by smooth metallic enclosure. The new approach makes use of a two-dimensional periodical array patterned on the top and bottom surfaces of the rectangular waveguide made by conventional printed circuit board (PCB) photolithographic fabrication process for proof of concept. Properly applying the slow-wave and high-impedance-surface behaviors of the twodimensional periodical structures to be presented in Section II, Section III describes the deembedding procedures to remove the effects of the mode converters necessary to interface the SRW to external measurement ports for $\mathrm{TE}_{10}, \mathrm{TM}_{00}$, and $\mathrm{TM}_{10}$ modes. The deembedded data are applied to validate the results obtained by the finite-element method (FEM). Section IV reports the newly synthesized $\mathrm{TE}_{10}$ mode rectangular waveguide with a cutoff frequency that is much lower than that of the all-metallic rectangular waveguide based on the same structural and material constants; the slow-wave factor of the $\mathrm{TE}_{10}$ mode SRW is also significantly greater than $\sqrt{\varepsilon_{r}}$, which is the theoretical limit of a conventional rectangular waveguide. $\varepsilon_{r}$ is the relative dielectric constant of the PCB substrate. One example employing the $\mathrm{TE}_{10}$ mode of the proposed SRW was the design of miniaturized four-slot antenna array in the $5 \mathrm{GHz}$ ISM band, demonstrating antenna gain of $4.28 \mathrm{dBi}, 60 \%$ area reduction in waveguide cross-section, measuring $71.0 \mathrm{~mm} \times 10.5 \mathrm{~mm} \times 0.9 \mathrm{~mm}$ (including the microstrip-to-waveguide transition) on a conventional FR4 $\left(\varepsilon_{r}=4.1\right)$ substrate [37]. Besides investigating in detail the $\mathrm{TE}_{10}$ mode propagation of the SRW, Section V investigates the propagations of $\mathrm{TM}_{00}$ and $\mathrm{TM}_{10}$ modes of the same SRW. Notably, no $\mathrm{TM}_{00}$ and $\mathrm{TM}_{10}$ modes can exist in the conventional rectangular waveguide with an all-metallic enclosure. Section VI concludes this paper.

\section{Synthetic Rectangular WaVeguide: Design AND OPERATIONAL PRINCIPLES}

\section{A. Dispersion Characteristics of Parallel-Coupled Electromagnetic Bandgap Surfaces}

Fig. 1(a) illustrates the conventional rectangular waveguide made using four smooth metal plates. If the top and bottom 

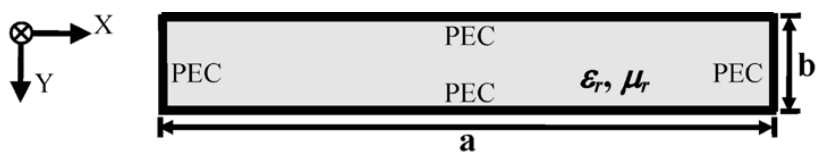

(a)
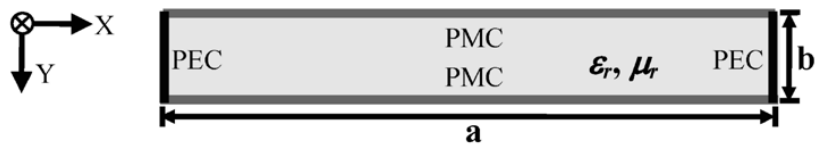

(b)

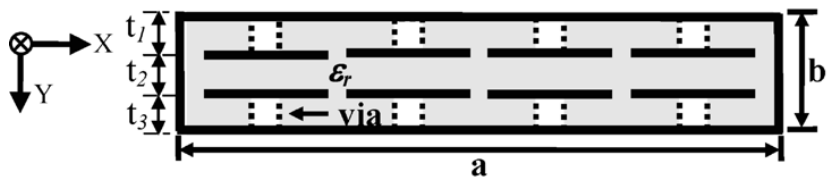

(c)

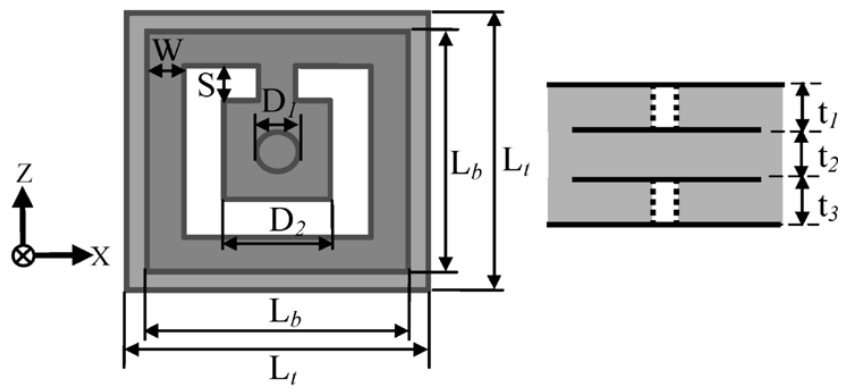

(d)

Fig. 1. Rectangular waveguide structures (a) using all-metallic enclosure: $\mathrm{a}=$ $7.0 \mathrm{~mm}, \mathrm{~b}=0.609 \mathrm{~mm}, \varepsilon_{r}=3.38, \tan \delta=0.0035$. (b) Top and bottom surfaces incorporating PMC. (c) Proposed SRW: $\mathrm{t}_{1}=t_{2}=t_{3}=0.203 \mathrm{~mm}$. (d) EBG unit cell applied in SRW: W $=0.2 \mathrm{~mm}, \mathrm{~S}=0.2 \mathrm{~mm}, D_{1}=$ $0.25 \mathrm{~mm}, \mathrm{D}_{2}=0.55 \mathrm{~mm}, \mathrm{~L}_{t}=1.55 \mathrm{~mm}, \mathrm{~L}_{b}=1.35 \mathrm{~mm}$.

metal plates are replaced by perfect magnetic conductors (PMCs) as shown in Fig. 1(b), the guiding characteristics of the conventional rectangular waveguide are drastically changed. This paper presents a new approach for designing integrated rectangular waveguides. As illustrated in Fig. 1(c), the integrated SRW consists of two vertical plated sidewalls and two EBG surfaces replacing the top and bottom plates of Fig. 1(a). The proposed SRW can support propagating modes in both rectangular waveguides as shown in Fig. 1(a) and (b). The SRW extensively applies the propagation characteristics of periodical structures.

In the SRW, the two-dimensional periodical array, or the so-called EBG surface, constitutes both top and bottom metallic surfaces of the conventional rectangular waveguide. Fig. 1(d) details the unit cell design of the EBG surface. The unit cell consists of a pair of connected spiral coils: one is located at the top surface and the other at the bottom surface. The connected spiral coils are dc-connected by a through-hole at the center. The spiral coils form coupled inductors, and the overlapped area between the spirals creates additional capacitances. Furthermore, inductive and capacitive couplings also exist between adjacent cells. Thus the propagation characteristics of the periodical structure are highly dispersive. The full-wave finite-element-based simulation package HFSS is applied to obtain the dispersion characteristics of the multilayered two-dimensional periodical structure of Fig. 1(d). In the numerical investigation, two pairs of master-slave boundaries of assumed phase differences were placed at four edges of the unit cell to compute a corresponding complex frequency of the eigenvalue, from which the phase and attenuation constants $(\gamma=\beta-j \alpha)$ of the two-dimensional periodical structure were obtained [38].

Perfect matching layers (PMLs) were placed adjacent to the top and bottom surfaces of the unit cell with to absorb the radiated waves. Fig. 2 plots the corresponding Brillouin diagram of the EBG cell. The straight solid (dashed) lines represent the propagation of the TEM mode in free-space (dielectric medium with relative permittivity of $\varepsilon_{r}$ ). These straight lines form two triangles, as shown in Fig. 2. The region outside the solid triangle belongs to the radiation zone. Inside the solid triangle, however, is the bound mode region. In this case study, predominant TEM solutions were observed inside the triangle formed by the dashed lines for operating frequencies below $9.85 \mathrm{GHz}$, manifesting the slow-wave factor greater than $\sqrt{\varepsilon_{r}}$, which is the upper ceiling for most conventional guided-wave structures. The electric (magnetic) fields are mostly perpendicular (parallel) to the spiral coils, and negligible field components are observed along the direction of propagation. Near zero operating frequency, the phase constant asymptotically approaches that of the TEM mode in the dielectric medium with $\varepsilon_{r}$. Near $9.85 \mathrm{GHz}$, the group velocity approaches zero. The magnitude of the modal currents near cutoff becomes much smaller than that of the predominant TEM mode at $5.0 \mathrm{GHz}$. Above $15 \mathrm{GHz}$, predominant TE mode solutions were observed, since electric fields exist only in the plane transverse to the direction of propagation. Thus the TE mode is designated as the first high-order mode of the periodical structure shown in Fig. 1(d). Below 9.85 $\mathrm{GHz}$, the EBG periodical structure is a slow-wave guiding structure, establishing the core operational principle for designing miniaturized integrated SRW.

Equally important, the Brillouin diagram shows a broad spectrum of forbidden band between 9.85 and $15.0 \mathrm{GHz}$. Such stopband connected to the high impedance surface, or the so-called magnetic surface, had been extensively studied [1], [17]. Thus the SRW of Fig. 1(c) seems to exhibit guiding properties closely resembling those of Fig. 1(b) in the forbidden band. In the passband below $9.85 \mathrm{GHz}$, however, the SRW is more like the conventional rectangular waveguide shown in Fig. 1(a).

\section{B. Simplified Rectangular Waveguide Models for SRW}

As mentioned above, EBG surfaces, which form both top and bottom layers of the SRW, behave like magnetic walls (electrical walls) in the stopband (passband). Therefore, Fig. 1(a) and (b) represent simplified rectangular waveguide models of the SRW. Since the proposed SRW is realizable by multilayered integrated circuit processes, the lateral dimensions (along the $x$-axis) of the SRW are typically much larger than the thickness of the substrate along the $y$-axis. Consequently, the lowest order TE modes of Fig. 1(a) are $\mathrm{TE}_{10}$ and $\mathrm{TE}_{20}$, etc., whereas the lowest TM modes of Fig. 1(b) are $\mathrm{TM}_{00}$ and $\mathrm{TM}_{10}$, etc. $\mathrm{TM}_{00}$ mode is essentially a TEM mode with uniform transverse $E_{x}$ and $H_{y}$ fields in the waveguide cross-section, which manifests the TEM mode waveguide as reported by Sievenpiper et al. [19], Itoh et al. [17], and Kildal et al. [18], respectively. The procedures for deriving the solutions of $\mathrm{TE}_{n 0}$ modes are well documented [39]. 


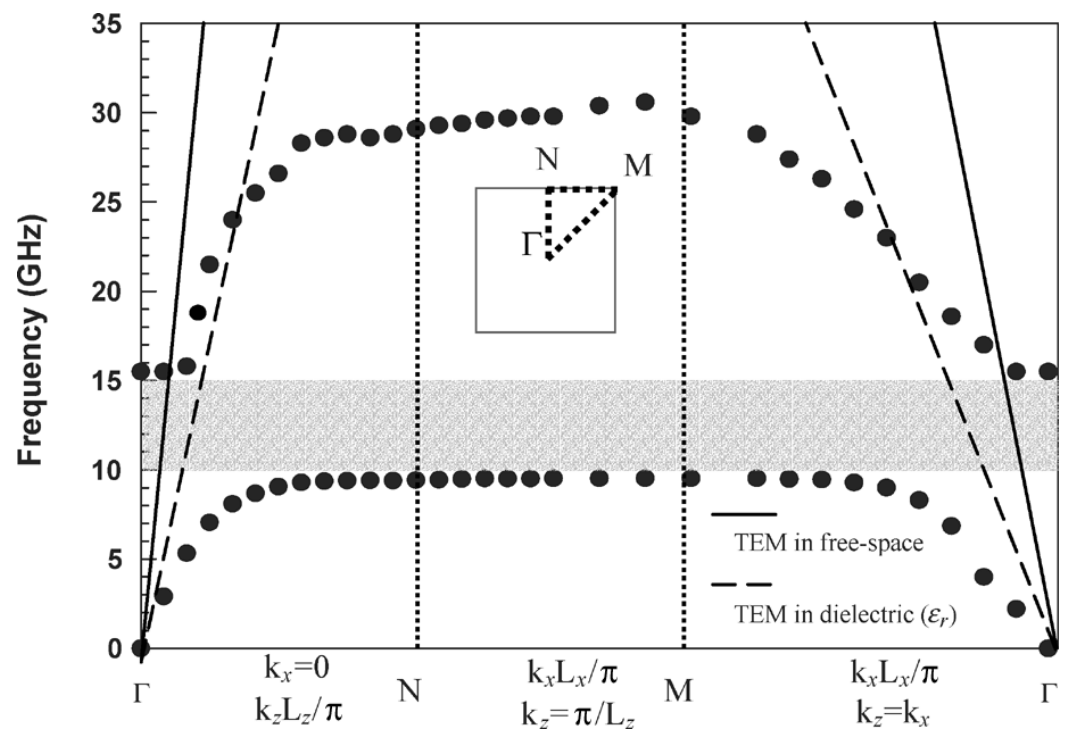

Fig. 2. Brillouin diagram of the two-dimensional periodical array made of unit cell of Fig. 1(d).

The same procedures are also applied to investigating the $\mathrm{TM}_{m 0}$ modes of idealized SRW.

For $\mathrm{TE}_{n 0}$ modes

$$
k=\frac{\omega}{c} \sqrt{\varepsilon_{r} \mu_{r}}
$$

where $k$ is the wave number of the dielectric medium with relative permittivity $\varepsilon_{r}$ and relative permeability $\mu_{r}$

$$
\beta_{n}=\sqrt{k^{2}-\left(\frac{n \pi}{a}\right)^{2}}, \quad n=1,2,3, \ldots
$$

where $n$ represents order of the TE mode and $a$ is the lateral dimension of SRW in $x$-direction. The lowest order mode is $\mathrm{TE}_{10}$. Only three field components exist for the $\mathrm{TE}_{n 0}$ mode, namely

$$
\begin{aligned}
E_{y} & =-j \omega \mu_{0} \mu_{r} a H_{0} \sin \left(\frac{n \pi}{a} x\right) e^{-j \beta_{n} z} \\
H_{x} & =j \beta a H_{0} \sin \left(\frac{n \pi}{a} x\right) e^{-j \beta_{n} z} \\
H_{z} & =H_{0} n \pi \cos \left(\frac{n \pi}{a} x\right) e^{-j \beta_{n} z} .
\end{aligned}
$$

The $\mathrm{TE}_{n 0}$ mode has a cutoff frequency strictly related to lateral dimension $a$, order $n$, and material constants, i.e.,

$$
f_{\text {cutoff }, \mathrm{TE}_{n 0}}=\frac{n c}{2 a \sqrt{\varepsilon_{r} \mu_{r}}} .
$$

For $\mathrm{TM}_{m 0}$ modes

$$
\beta_{m}=\sqrt{k^{2}-\left(\frac{m \pi}{a}\right)^{2}}, \quad m=0,1,2, \ldots
$$

where $m$ represents order of the TM mode and $a$ is the lateral dimension of SRW in $x$-direction. The lowest order mode is $\mathrm{TM}_{00}$, which is the zero-cutoff limit of $\mathrm{TM}_{m 0}$ modes. Only three field components exist for the $\mathrm{TM}_{m 0}$ mode, namely

$$
\begin{aligned}
H_{y} & =-j \omega \varepsilon_{0} \varepsilon_{r} a E_{0} \cos \left(\frac{m \pi}{a} x\right) e^{-j \beta_{m} z} \\
E_{x} & =-j \beta_{m} a E_{0} \cos \left(\frac{m \pi}{a} x\right) e^{-j \beta_{m} z} \\
E_{z} & =E_{0} m \pi \sin \left(\frac{m \pi}{a} x\right) e^{-j \beta_{m} z} .
\end{aligned}
$$

The $\mathrm{TM}_{m 0}$ mode has a cutoff frequency strictly related to lateral dimension $a$, order $m$, and material constants, i.e.,

$$
f_{\text {cutoff, }} \mathbf{T M}_{m 0}=\frac{m c}{2 a \sqrt{\varepsilon_{r} \mu_{r}}} .
$$

It is interesting to notice that the interchange of $E$ and $H$, sine and cosine leads to the interchange of $\mathrm{TE}_{n 0}$ and $\mathrm{TM}_{m 0}$ modes. Additionally, the SRW shows different dispersion characteristics from those of idealized $\mathrm{TE}_{n 0}$ and $\mathrm{TM}_{m 0}$ modes. In later sections, we will show that the $\mathrm{TM}_{00}$ mode of the SRW has a cutoff frequency and that both $\mathrm{TE}_{10}$ and $\mathrm{TM}_{10}$ modes have different cutoff frequencies, not dictated by (6) and (11).

\section{Synthetic Rectangular WAVEguide: WAVEguide TRANSITIONS TO PLANAR TRANSMISSION LINES AND DEEMBEDDING STUDIES}

\section{A. Integrated Synthetic Rectangular Waveguide}

As shown in Fig. 1(c), the SRW was formed on an RO4003 PCB with a relative dielectric constant of 3.38 and a $\tan \delta$ of 0.0035 . The metal printed on the substrate was copper with a thickness of $17 \mu \mathrm{m}$. Notably, the EBG surface comprised four unit cells in the transverse direction of the SRW. Similar design had been reported and applied in designing the dual-band leakymode antenna [7], indicating that the design of the EBG surface shown in Fig. 1(c) has stopband characteristics similar to those two-dimensional arrays of infinite number of cells.

The propagating energy of the SRW must be interfaced to planar guiding structures to become a useful integrated guiding device. This section describes various designs to interface SRW supporting $\mathrm{TE}_{10}, \mathrm{TM}_{00}$, and $\mathrm{TM}_{10}$ modes. A streamline mode converter made of a tapered microstrip has been successfully demonstrated as a good waveguide transition device to interface a microstrip line and a rectangular waveguide supporting $\mathrm{TE}_{10}$ mode [40]-[44]. The $E$-field of this tapered microstrip mode resides mostly in the transverse $y$-direction in the same way the $\mathrm{TE}_{10}$ mode does, thus ensuring smooth field transition in the mode converter. Fig. 3(a)-(c) shows the top, front, and side 


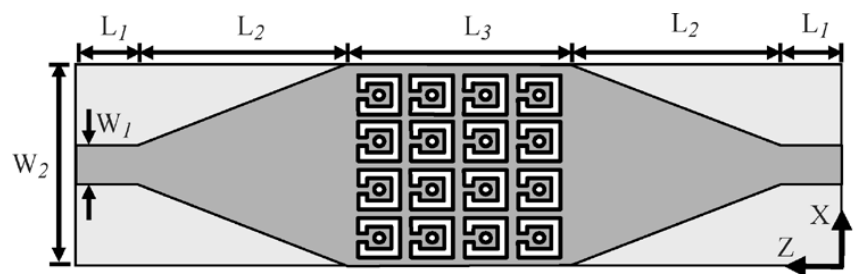

(a)

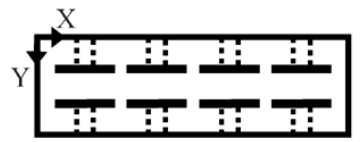

(b)

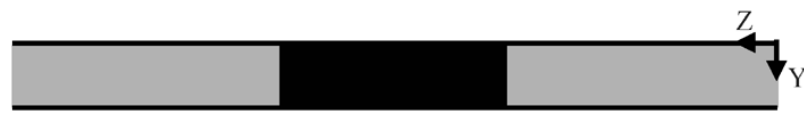

(c)

Fig. 3. $\mathrm{TE}_{10}$ mode SRW including microstrip-to-waveguide transitions. (a) Top view: $\mathrm{L}_{1}=3 \mathrm{~mm}, \mathrm{~L}_{2}=8 \mathrm{~mm}, \mathrm{~L}_{3}=7.2 \mathrm{~mm}, \mathrm{~W}_{1}=0.8 \mathrm{~mm}, \mathrm{~W}_{2}=$ $7 \mathrm{~mm}$. (b) Front view: waveguide thickness $(y-$ axis $)=0.609 \mathrm{~mm}$. (c) Side view.

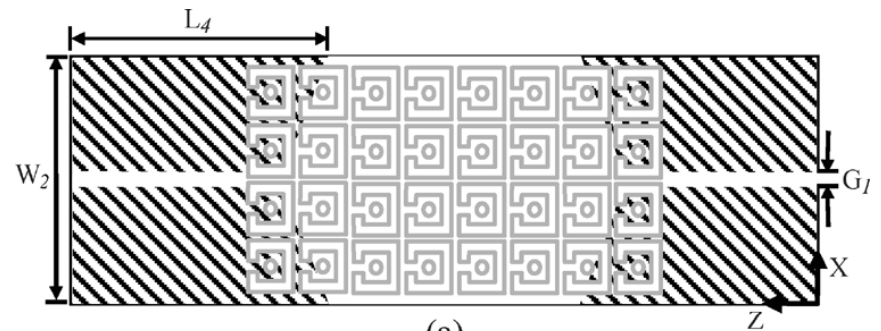

(a)

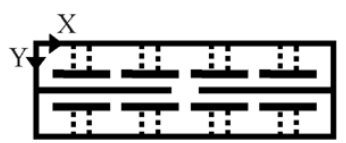

(b)

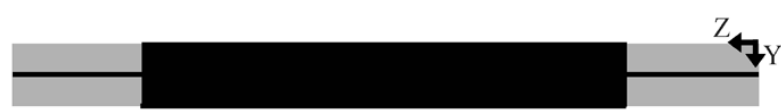

(c)

Fig. 4. $\mathrm{TM}_{00}$ mode SRW including slotline-to-waveguide transitions. (a) Top view: $\mathrm{G}_{1}=0.15 \mathrm{~mm}, \mathrm{~L}_{4}=13.05 \mathrm{~mm}$. (b) Front view: waveguide thickness $(y-$ axis $)=0.609 \mathrm{~mm}$. (c) Side view.

views of a back-to-back connected SRW supporting $\mathrm{TE}_{10}$ mode with interfaces to two external microstrip ports.

Resorting to (9), the $\mathrm{TM}_{00}$ mode exhibits a constant $E$-field polarization in the $x$-direction, suggesting a symmetric PEC plane at $x=a / 2$. Therefore, a tapered finline inserted on the horizontal plane of $y=b / 2$ is proposed for the $\mathrm{TM}_{00} \mathrm{SRW}$ waveguide transition as illustrated in Fig. 4(a)-(c). Likewise a tapered finline is the waveguide transition for $\mathrm{TM}_{00}$ mode SRW. Fig. 5 illustrates how a tapered coplanar waveguide (CPW), which is symmetrical about a PMC surface at $x=a / 2$, works as a $\mathrm{TM}_{10}$ mode converter.

\section{B. Single-Mode Approximate Algorithm for Deembedding Integrated $S R W$}

As mentioned above, the integrated SRW consists of the SRW and additional waveguide transitions. The effects of mode con-

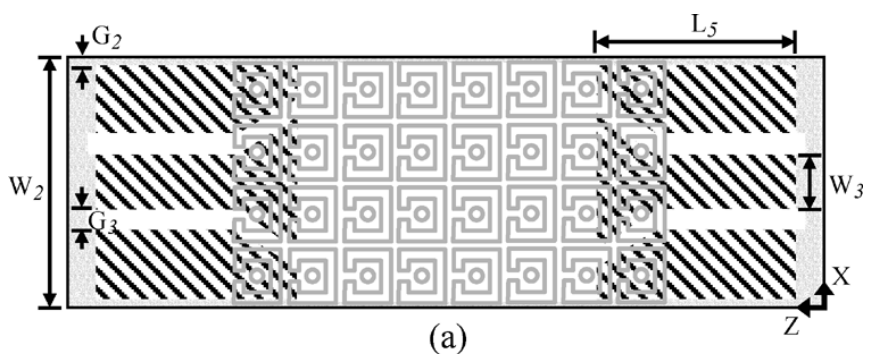

(a)

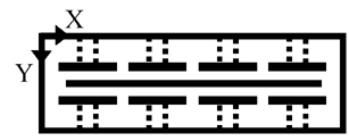

(b)

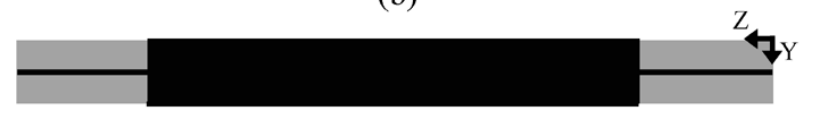

(c)

Fig. 5. $\mathrm{TM}_{10}$ mode $\mathrm{SRW}$ including CPW-to-waveguide transitions. (a) Top view: $\mathrm{G}_{2}=0.425 \mathrm{~mm}, \mathrm{G}_{3}=0.7 \mathrm{~mm}, \mathrm{~L}_{5}=12.85 \mathrm{~mm}, \mathrm{~W}_{3}=0.75 \mathrm{~mm}$ (b) Front view: waveguide thickness $(y-$ axis $)=0.609 \mathrm{~mm}$. (c) Side view.

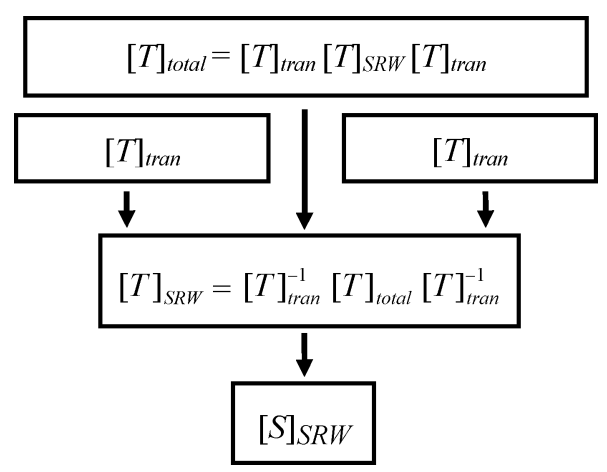

Fig. 6. Proposed deembedding procedures for extracting guiding characteristics of the planar rectangular waveguide.

verters must be deembedded to obtain the propagation characteristics of the SRW out of two-port scattering analyses or measurements. Herein a single-mode approximation as depicted in Fig. 6 is presented.

The overall measured/theoretical transmission matrix $[T]_{\text {total }}$ of the integrated SRW, including two mode converters and the SRW, was obtained by converting the measured/theoretical $S$-parameters to $T$-parameters [45]. The measurement was conducted after the short-open-load-through calibration procedures that had been performed by the HP8510C vector network analyzer. The theoretical analysis of the test device, however, was based on HFSS full-wave scattering simulation. Next, the $[T]_{\operatorname{tran}}$ of the mode converter was obtained theoretically by HFSS based on the single-mode approximation by assuming microstrip, finline, and coplanar waveguide mode of propagation for $\mathrm{TE}_{10}, \mathrm{TM}_{00}$, and $\mathrm{TM}_{10}$, respectively. Then the $[T]_{\text {SRW }}$ was obtained by pre- and post-multiplying $[T]_{\text {total }}$ by the inverse of $[T]_{\text {tran }}$. Finally, $[S]_{\text {SRW }}$ was obtained by simply converting the corresponding $[T]_{\mathrm{SRW}}$ into the $[S]_{\text {SRW }}$.

To validate the deembedding procedure of Fig. 6, conventional $\mathrm{TE}_{10}$ mode rectangular waveguide with the structural and 


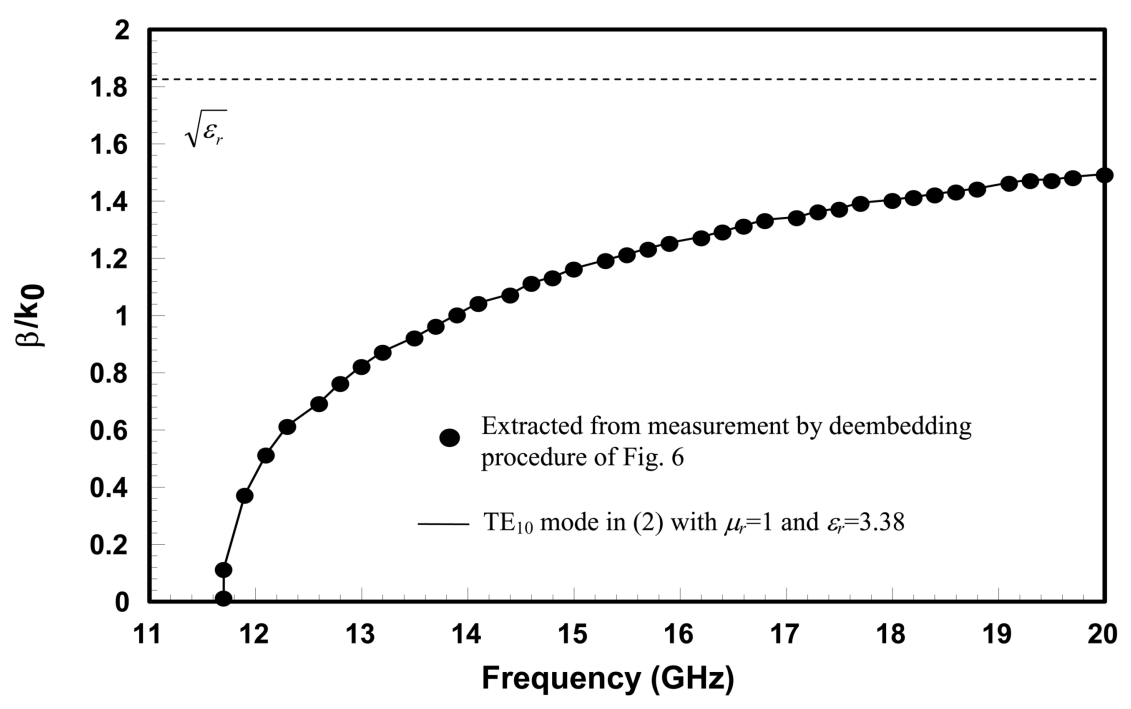

Fig. 7. Comparison of the extracted normalized phase constants of the conventional rectangular waveguide with those obtained by exact equation.

material constants shown in Fig. 1(a) and tapered microstrip mode converter shown in Fig. 3 were investigated. Initially, the two-port scattering parameters of the end-to-end integrated rectangular waveguide obtained by measurement and by HFSS simulation were compared. Both data show excellent agreement in magnitude and phase. Then, the measured overall two-port scattering parameters were deembedded to obtain the complex propagation constant of the conventional rectangular waveguide. Fig. 7 compares the extracted results with those obtained (2), showing excellent agreement between the two data sets. Therefore we conclude that the single-mode approximation assumed in the deembedding procedure of Fig. 6 is a good approximation for deembedding the integrated $\mathrm{TE}_{10}$ mode SRW as shown in Fig. 3.

In Sections IV and V, the single-mode approximate deembedding procedure is applied to recover the complex propagation constant of the integrated SRW supporting $\mathrm{TE}_{10}, \mathrm{TM}_{00}$, and $\mathrm{TM}_{10}$ modes. The $Q$-factor of the conventional rectangular waveguide was also investigated using the basic theory of a rectangular waveguide cavity [45]. The total $Q$-factor of the conventional rectangular waveguide is the inverse of the summation of $1 / Q_{\mathrm{d}}$ and $1 / Q_{\mathrm{c}}$, where $Q_{\mathrm{d}}$ and $Q_{\mathrm{c}}$ are the $Q$-factors of the rectangular waveguide with dielectric loss and conductor loss, respectively. The conducting walls, having high conductivity and a thickness of $17 \mu \mathrm{m}$, more than five times the skin depths above the cutoff frequency, were applied to realize a conventional rectangular waveguide, whose $Q_{\mathrm{c}}$ is about 9650 , between 12 and $20 \mathrm{GHz}$. However, $Q_{\mathrm{d}}$, which is proportional to the inverse of the $\tan \delta$ value of the substrate in the rectangular waveguide, is about 285.7, dominating the power dissipation in the rectangular waveguide. Therefore, the theoretical $Q$-factor of the conventional rectangular is about 277 . However, the measured $Q$-factor, extracted from the deembedded propagation constant of the conventional rectangular waveguide, without the effects of the mode converters, is about 275 between 12 and $20 \mathrm{GHz}$, indicating close agreement between the measured results and the theoretical predictions.

\section{TE $_{10}$ Mode Synthetic Rectangular Waveguide (SRW)}

Parallel to obtaining the dispersion diagram of two-dimensional periodical structure made of unit cells shown in Fig. 1(d), the master-slave boundaries along the z-direction (longitudinal axis) of Fig. 1(c) were applied to solve the complex propagation constant for the SRW. Measured two-port scattering parameters of the integrated $\mathrm{TE}_{10}$ mode SRW shown in Fig. 3 were also obtained; then the deembedding procedure of Fig. 6 was invoked. Fig. 8 compares the normalized phase constants (slow-wave factors) obtained by two approaches, showing excellent agreement between the extracted measured data and theoretical results. The cutoff frequency of the $\mathrm{TE}_{10}$ mode SRW was almost $4.10 \mathrm{GHz}$, conventional waveguide with the same outer dimensions and material constant. The slow-wave factor of the $\mathrm{TE}_{10}$ mode SRW rises quickly to the theoretical limit $\sqrt{\varepsilon_{r}}$ at 4.38 $\mathrm{GHz}$ from the cutoff frequency. Then the slow-wave factor ascends almost linearly to 4.35 at approximately $5.78 \mathrm{GHz}$. Then it levels off to 4.9 at $6.85 \mathrm{GHz}$. Meanwhile, the deembedded $Q$-factor is about 260 , representing only $5.8 \%$ degradation in $Q$-factor as compared to the conventional waveguide although it operates at much higher frequency. Notably, a conventional rectangular waveguide with the same cutoff frequency using the same dielectric material and thickness as the SRW would have lateral dimension of 19.9 against $7.0 \mathrm{~mm}$ in the SRW design. If the conventional rectangular waveguide has slow-wave factor of $\sqrt{\varepsilon_{r}}$, approaching the theoretical limit immediately produces an estimated area reduction of $6.7(85 \%)$ (at $5.78 \mathrm{GHz}$ ) using the particular SRW design. The results clearly demonstrate that the proposed SRW is ideal for miniaturized MIC design, which requires a high- $Q$ transmission line.

In obtaining the complex propagation constant of the $\mathrm{TE}_{10}$ mode SRW using HFSS simulation, only three field components in the SRW were observed. Fig. 9(a)-(c) plots the transverse $E_{y}$, transverse $H_{x}$, and longitudinal $H_{z}$ field components at $6 \mathrm{GHz}$, respectively. The value $E_{y}$ and $H_{x}$ [Fig. 9(a) and 


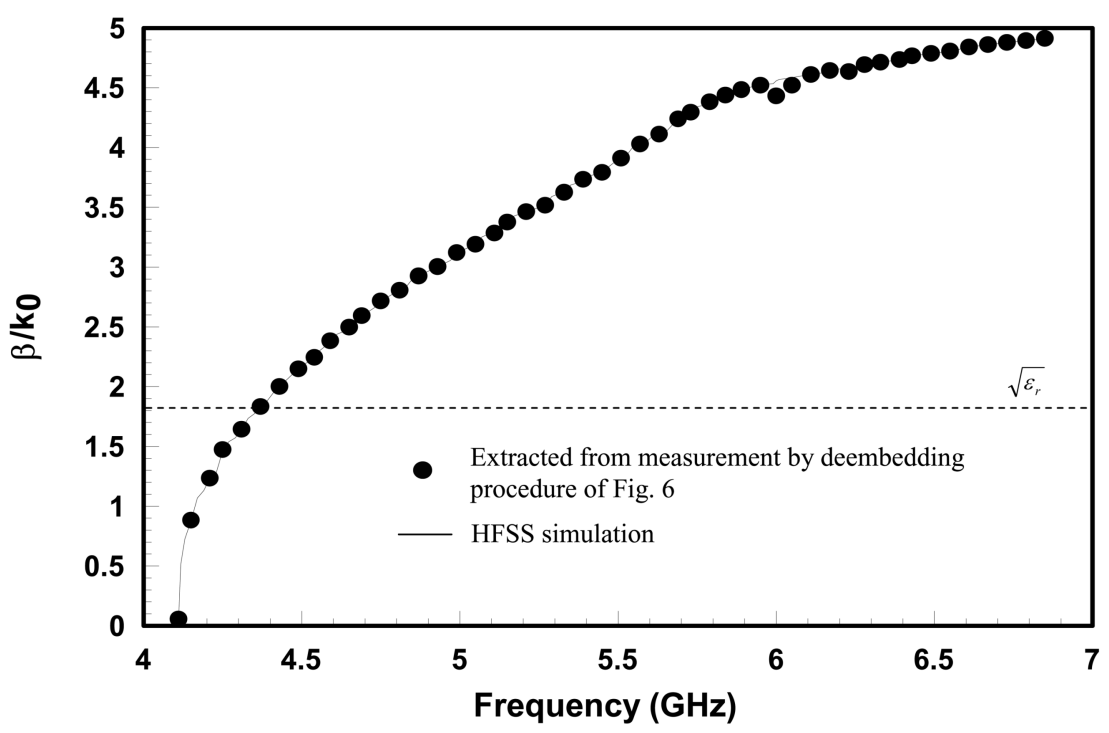

Fig. 8. Comparison of the extracted normalized phase constants of the $\mathrm{TE}_{10}$ mode SRW with those obtained by HFSS simulation.

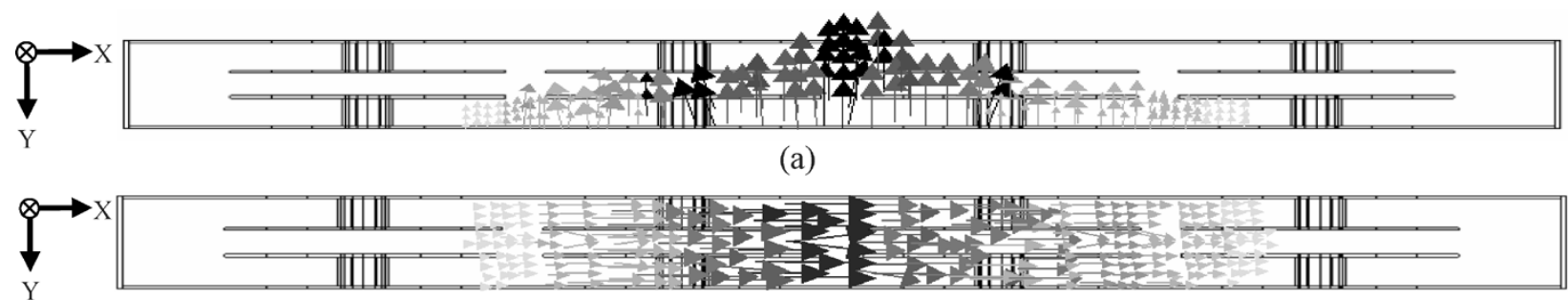

(b)

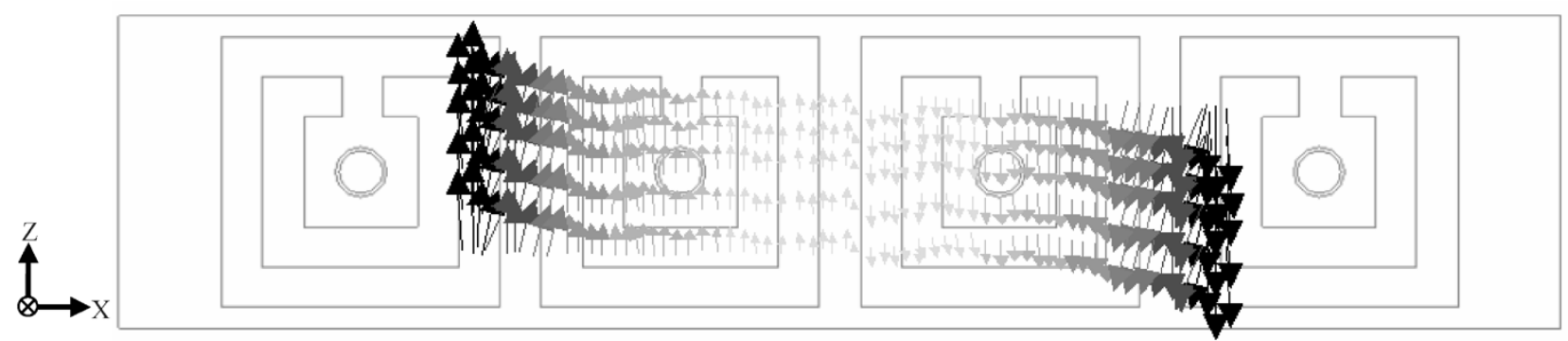

(c)

Fig. 9. Field distribution of the synthetic $\mathrm{TE}_{10}$ made rectangular waveguide at $6.0 \mathrm{GHz}$. (a) Two-dimensional (2-D) $E_{y}$-field, (b) 2-D $H_{x}$-field, and (c) 2-D $H_{z}$-field.

Fig. 9(b)] approximately follow the sine distribution and $H_{z}$ [Fig. 9(c)] the cosine distribution. Such observations agree well with the field distributions of an ideal $\mathrm{TE}_{10}$ mode waveguide governed by (3)-(5). Thus the results shown in Fig. 8 validate the propagation of the $\mathrm{TE}_{10}$ mode in the SRW as shown in Fig. 3.

\section{V. $\mathrm{TM}_{00}$ AND $\mathrm{TM}_{10}$ Mode Synthetic RECTANGUlar WAVEGUIDES (SRWs)}

The making of $\mathrm{TM}_{00}$ and $\mathrm{TM}_{10}$ SRW as illustrated in Figs. 4 and 5 is beyond our current capability. Thus two theoretical methods were applied to confirm that the $\mathrm{TM}_{00}$ and $\mathrm{TM}_{10}$ modes exist in the proposed SRW. These methods are the deembedding procedure of Fig. 6 and FEM using HFSS.

Fig. 10(a) and (b) investigates the through characteristics of the back-to-back connected SRW supporting $\mathrm{TM}_{00}$ and $\mathrm{TM}_{10}$ modes, respectively. The $3 \mathrm{~dB}$ bandwidth is between 10.83 and $11.8 \mathrm{GHz}\left(11.1\right.$ and $12.2 \mathrm{GHz}$ ) for the integrated $\mathrm{TM}_{00}$ mode $\left(\mathrm{TM}_{10}\right.$ mode) SRW, as shown in Figs. 4 and 5. Both transmission regions mentioned above, however, fall into the stopband region of Fig. 2, implying that the EBG surfaces behave like magnetic surfaces. Since the transmission losses are relatively high and the bandwidths are relatively narrow, the single-mode approximate deembedding procedure may not be accurate. Nevertheless, the deembedding method can generate an estimate of the SRW phase constant.

Fig. 11 shows the superimposed plots of the normalized phase constants obtained by the deembedding procedure and HFSS simulation, respectively. The curves with solid and hollow square symbols represent the $\mathrm{TM}_{00}$ mode solutions obtained by deembedding and HFSS. Data below 10.4 and above $12.3 \mathrm{GHz}$ are not shown for the deembedding case, since the deembedding phase constant changed drastically. Between 


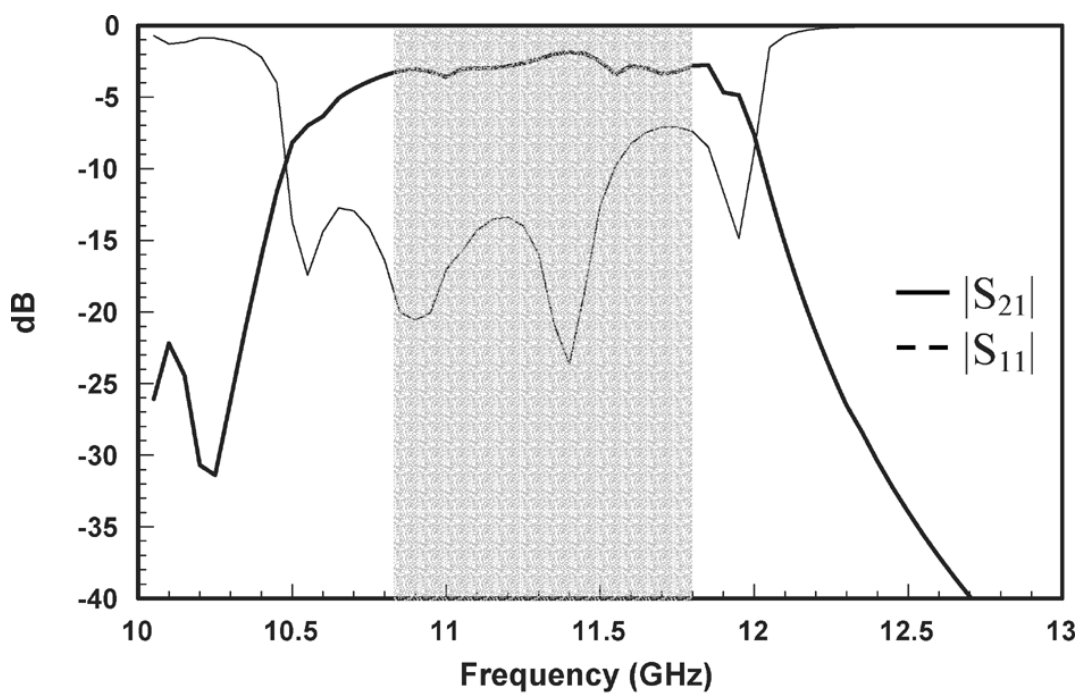

(a)

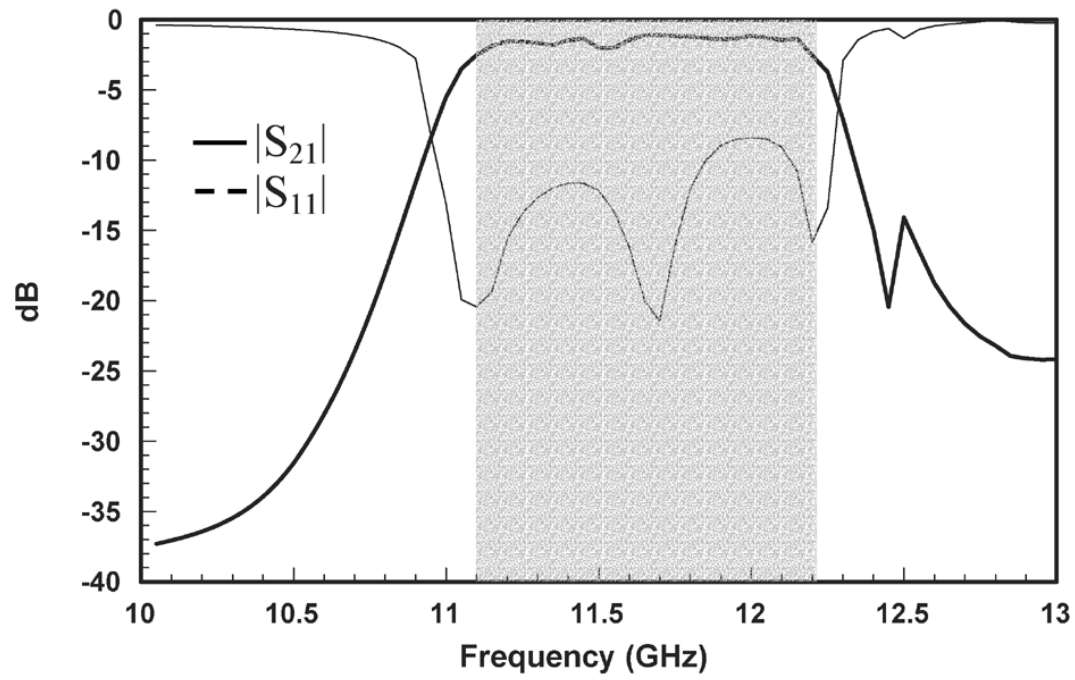

(b)

Fig. 10. Scattering analyses of the integrated SRW with various waveguide transitions. (a) $\mathrm{TM}_{00}$ mode converter and (b) TM 10 mode converter.

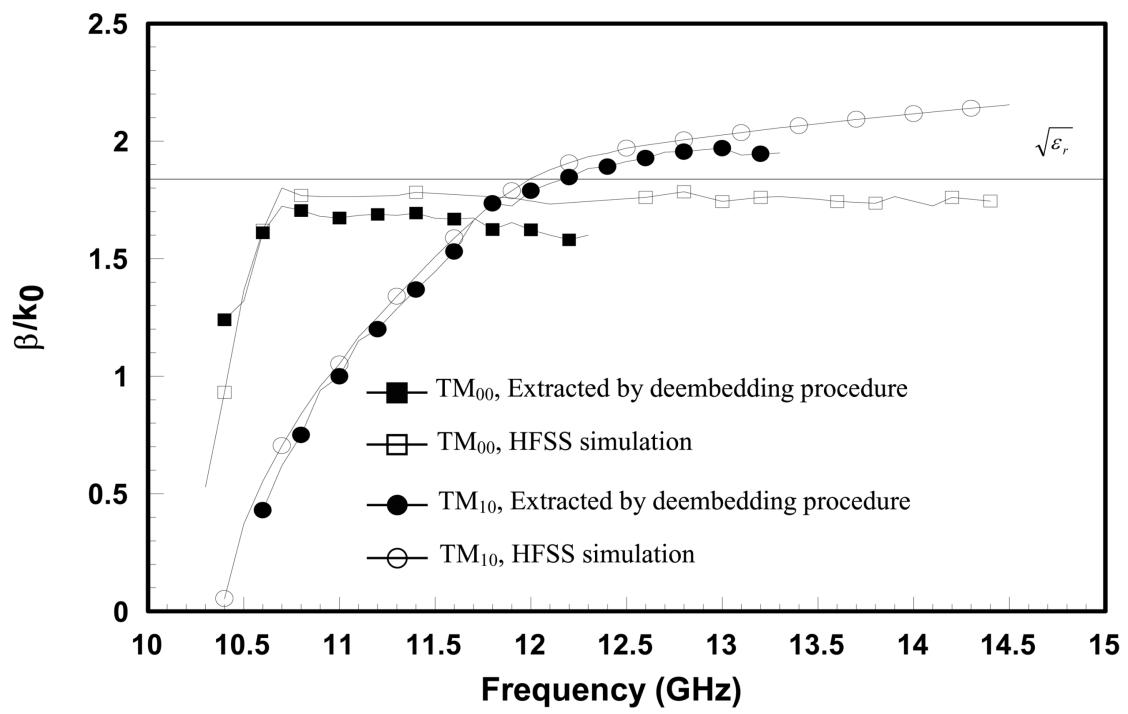

Fig. 11. Normalized phase constants of the proposed synthetic $\mathrm{TM}_{00}$ and $\mathrm{TM}_{10}$ mode rectangular waveguides.

10.8 and $11.8 \mathrm{GHz}$, the deembedding phase constant was about $6.2 \%$ lower than that obtained by HFSS. It is interesting to note that the deembedded phase constant is very close to the HFSS data between 10.5 and $10.6 \mathrm{GHz}$. 


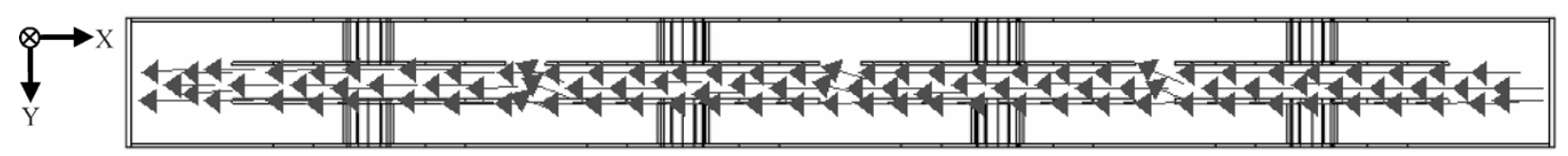

(a)

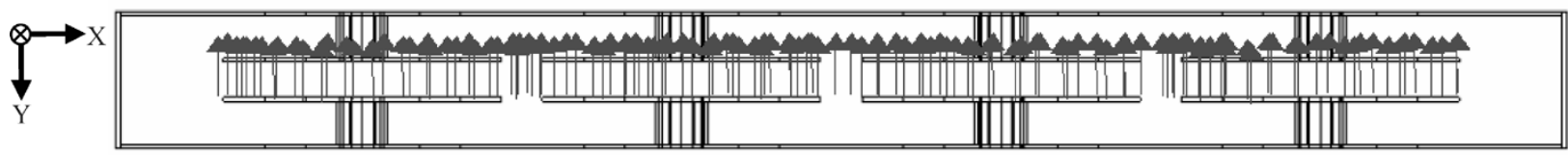

(b)

Fig. 12. Field distribution of the synthetic $\mathrm{TM}_{00}$ mode SRW at $11.0 \mathrm{GHz}$. (a) 2-D $E_{x}$-field and (b) 2-D $H_{y}$-field.

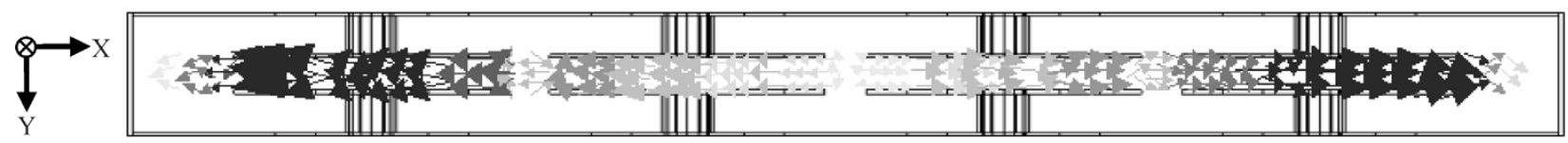

(a)

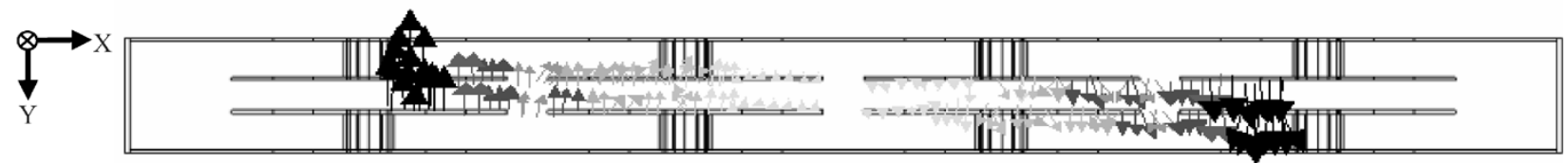

(b)

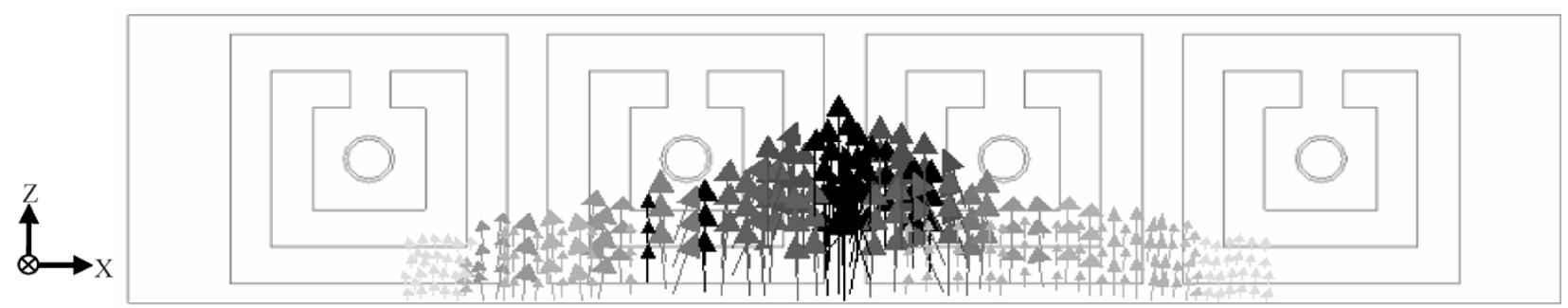

(c)

Fig. 13. Field distribution of the synthetic $\mathrm{TM}_{10}$ mode rectangular waveguide at $11.4 \mathrm{GHz}$. (a) 2-D $E_{x}$-field, (b) 2-D $H_{y}$-field, and (c) 2-D $E_{z}$-field.

TABLE I

Theoretical $Q$-Factor of THE TM TM $_{00}$ Mode SRW at $11.0 \mathrm{GHz}$

\begin{tabular}{c|l|c|c}
\hline & \multicolumn{1}{|c|}{$\tan \delta$} & $\sigma$ & $Q$-factor \\
\hline 1 & 0.0 & PEC & 664 \\
\hline 2 & 0.0035 & PEC & 199 \\
\hline 3 & 0.0 & $5.813 \times 10^{7}$ & 548 \\
\hline 4 & 0.0035 & $5.813 \times 10^{7}$ & 187 \\
\hline \multicolumn{4}{|c}{$\sigma:$ conductivity, $\tan \delta:$ loss tangent } \\
\hline
\end{tabular}

The slow-wave factor of $\mathrm{TM}_{00}$ mode also rises sharply from the cutoff frequency near $10.2 \mathrm{GHz}$ and approaches but never exceeds the theoretical limit $\sqrt{\varepsilon_{r}}$. After reaching $\sqrt{\varepsilon_{r}}$ at 10.6 $\mathrm{GHz}$, the slow-wave factor flattens and approaches to 14.5 $\mathrm{GHz}$, beyond which HFSS fails to produce stable data. When preparing the $\mathrm{TM}_{00}$ mode data obtained by HFSS up to 14.5 $\mathrm{GHz}$, the modal field distributions were plotted and confirmed to be governed by (8)-(10) for $m=0$. Fig. 12 shows the field distribution at $11.0 \mathrm{GHz}$. Only two field components $E_{x}$ and $H_{y}$ were observed, and field distributions, which exist only in region between two inner EBG surfaces, were keep uniform along the $x$-axis. Also, the transverse magnetic field $\left(H_{y}\right)$ shown in Fig. 12(b) was observed to be perpendicular to the EBG surfaces. Meanwhile the transverse electric field $\left(E_{x}\right)$ as shown in Fig. 12(a) is parallel to the EBG surfaces. Such ob-
TABLE II

Theoretical $Q$-Factor of THE TM 10 Mode SRW at $11.4 \mathrm{GHz}$

\begin{tabular}{c|l|c|c}
\hline & \multicolumn{1}{|c|}{$\tan \delta$} & $\sigma$ & $Q$-factor \\
\hline 1 & 0.0 & PEC & 673 \\
\hline 2 & 0.0035 & PEC & 192 \\
\hline 3 & 0.0 & $5.813 \times 10^{7}$ & 555 \\
\hline 4 & 0.0035 & $5.813 \times 10^{7}$ & 188 \\
\hline \multicolumn{3}{|c}{$\sigma:$ conductivity, $\tan \delta:$ loss tangent } \\
\hline
\end{tabular}

servations, which reveal the field distributions of pseudo-TEM mode in the SRW, agree with the ideal $\mathrm{TM}_{00}$ waveguide model shown in Fig. 1(b), confirming that the EBG surfaces behave like the PMC.

The same investigation and precautions were exercised when plotting the $\mathrm{TM}_{10}$ mode data. The curves with solid and hollow dots represent the $\mathrm{TM}_{10}$ mode solutions obtained by deembedding and HFSS, respectively. The deembedding phase constant was about $4.3 \%$ lower than that obtained by the HFSS data between 10.5 and $10.6 \mathrm{GHz}$. The slow-wave factor of $\mathrm{TM}_{10}$ mode approaches zero from the cutoff frequency near 10.4 $\mathrm{GHz}$ and exceeds the $\sqrt{\varepsilon_{r}}$ at $11.98 \mathrm{GHz}$. Then it levels off to 2.2 at $14.5 \mathrm{GHz}$. Fig. 13 shows FEM modal field distribution at $11.4 \mathrm{GHz}$. Only three field components $E_{x}, H_{y}$, and $E_{z}$ were observed. Notably, Fig. 13(a) and (b) reveals that 
the transverse field components $E_{x}$ and $H_{y}$ follow $\cos (\pi x / a)$ distributions. In the longitudinal direction, only the $E_{z}$ component exists, following $\sin (\pi x / a)$ distribution, thus verifying the $\mathrm{TM}_{10}$ mode solution.

The extracted $Q$-factors of the $\mathrm{TM}_{00}$ and $\mathrm{TM}_{10}$ mode SRWs were evaluated using HFSS. A series of numerical analyses were performed with different material constant values, including conductivity and loss tangent, to investigate the loss of SRW during the $\mathrm{TM}_{00}$ mode and $\mathrm{TM}_{10}$ mode operations. The $Q$-factor of the SRW without conductor loss and dielectric loss is about 664 (673) for $\mathrm{TM}_{00}$ mode $\left(\mathrm{TM}_{10}\right.$ mode), yielding the intrinsic loss of the SRW. Additionally, $Q$-factors with either conductor loss or dielectric loss are approximately 548 and 199 for $\mathrm{TM}_{00}$ mode and 555 and 192 for $\mathrm{TM}_{10}$ mode, respectively. The total $Q$-factors of $\mathrm{TM}_{00}$ and $\mathrm{TM}_{10}$ mode are approximately 187 and 188 , respectively. Notably, the total $Q$-factors of the $\mathrm{TM}_{00}$ and $\mathrm{TM}_{10}$ mode SRW are higher than that of a microstrip line on the same substrate, indicating that the synthetic integrated $\mathrm{TM}_{00}$ and $\mathrm{TM}_{10}$ mode waveguides have useful applications in MIC and antenna designs.

\section{CONCLUSION}

This paper designs and demonstrates the effectiveness of an SRW whose top and bottom surfaces are replaced with two-dimensional periodical structures. The proposed SRW is fabricated using conventional multilayered PCB technology and can be integrated with other planar circuits using various waveguide mode converters. The two-dimensional periodical structures comprise unit cells made of coupled coils; a slow-wave region for the lowest band and a stopband region above the slow-wave region. This paper demonstrates both theoretically and experimentally that combining the two distinct regions of propagation of two-dimensional periodical structures leads to the design of an SRW that simultaneously exhibits the following unique characteristics. First, the slow-wave factor of the particular SRW is 4.9 , which significantly exceeds the theoretical limit of $\sqrt{\varepsilon_{r}}$ for the conventional metallic rectangular waveguide in the $\mathrm{TE}_{10}$ mode. Second, the $Q$-factor is high: 260 for the $\mathrm{TE}_{10}$ mode, 187 for the $\mathrm{TM}_{00}$ mode, and 188 for $\mathrm{TM}_{10}$ mode for the particular case study. Third, the waveguide transitions, including the tapered microstrip, finline, and tapered CPW, are integrated with the SRW in the same polymer substrate for the synthetic $\mathrm{TE}_{10}, \mathrm{TM}_{00}$, and $\mathrm{TM}_{10}$ mode converters, respectively. Therefore, the proposed SRW is well suited to high-performance MIC designs and antenna array development.

\section{REFERENCES}

[1] D. Sievenpiper, L. Zhang, R. F. Jimenez Broas, N. G. Alexópolous, and E. Yablonovitch, "High-impedance electromagnetic surfaces with a forbidden frequency band," IEEE Trans. Microwave Theory Tech., vol. 47, no. 11, pp. 2059-2074, Nov. 1999.

[2] R. Coccioli, F.-R. Yang, K.-P. Ma, and T. Roh, "Aperature-coupled patch antenna on UC-PBG substrate," IEEE Trans. Microwave Theory Tech., vol. 47, no. 11, pp. 2123-2130, Nov. 1999.

[3] J. D. Shumpert, W. J. Chappell, and L. P. B. Katehi, "Parallel-plate mode reduction in conductor-backed slots using electromagnetic bandgap substrates," IEEE Trans. Microwave Theory Tech., vol. 47, no. 11, pp. 2099-2104, Nov. 1999.
[4] S. P. Skobelev and P.-S. Kildal, "Analysis of a hard strip-loaded conical horn by the method of generalized scattering matrices," IEEE Trans. Antennas Propag., vol. 51, no. 10, pp. 2918-2925, Oct. 2003.

[5] S. Maci, R. Magliacani, and A. Cucini, "Leaky-wave antennas realized by using artificial surfaces," in Proc. IEEE Antennas Propag. Int. Symp., 2003, pp. 1099-1102.

[6] K.-C. Chen, Y. Qian, C.-K. C. Tzuang, and T. Itoh, "A periodic microstrip radial antenna array with a conical beam," IEEE Trans. Antennas Propag., vol. 51, no. 4, pp. 756-765, Apr. 2003.

[7] Y.-C. Chen, C.-K. Wu, and C.-K. C. Tzuang, "Dual-frequency electricmagnetic-electric microstrip leaky-mode antenna of a single fan beam," IEEE Trans. Microwave Theory Tech., vol. 50, no. 12, pp. 2713-2720, Dec. 2002.

[8] C.-K. Wu, Y.-C. Chen, and C.-K. C. Tzuang, "Compressed-width leaky $\mathrm{EH}_{1}$ mode PBG antenna," IEEE Microwave Wireless Comp. Lett., vol. 13, no. 8, pp. 343-344, Aug. 2003.

[9] D. F. Sievenpiper, J. H. Schaffner, H. Jae Song, R. Y. Loo, and G. Tangonan, "Two-dimensional beam steering using an electrically tunable impedance surface," IEEE Trans. Antennas Propag., vol. 51, no. 10, pp. 2713-2722, Oct. 2003.

[10] C.-Y. Tsai and C.-K. C. Tzuang, "Applying electric-magnetic-electric (EME) composite metal strips to reduce the size of path antennas," in 2001 Asia-Pacific Microwave Conf. Dig., 2001, pp. 1151-1154.

[11] S. Pioch and J.-M. Laheurte, "Size reduction of microstrip antennas by means of periodic metallic patterns," Electron. Lett., vol. 39, no. 13, pp. 959-961, Jun. 2003.

[12] F.-R. Yang, K.-P. Ma, Y. Qian, and T. Itoh, "A uniplanar compact photonic-bandgap (UC-PBG) structure and its applications for microwave circuits," IEEE Trans. Microwave Theory Tech., vol. 47, no. 8, pp. 1509-1514, Aug. 1999.

[13] X. Gong, W. J. Chappell, and L. P. B. Katehi, "Reduced size capacitive defect EBG resonators," in 2002 IEEE MTT-S Int. Microwave Symp. Dig., 2002, pp. 1091-1094.

[14] D. Deslandes and K. Wu, "Single-substrate integration technique of planar circuits and waveguide filters," IEEE Trans. Microwave Theory Tech., vol. 51, no. 2, pp. 593-596, Feb. 2003.

[15] H. Xin, A. Higgins, J. Hacker, M. Kim, and M. Rosker, "Electromagnetic crystal (EMXT) waveguide band-stop filter," IEEE Microwave Wireless Comp. Lett., vol. 13, no. 3, pp. 108-110, Mar. 2003.

[16] H. Xin, A. Higgis, and M. Kim, "Tunable millimeter-wave band-stop filter using electromagnetic crystal (EMXT) surfaces," in 2003 IEEE AP-S Int. Microwave Symp. Dig., 2003, pp. 1107-1110.

[17] F.-R. Yang, K.-P. Ma, Y. Qian, and T. Itoh, "A novel TEM waveguide using uniplanar compact photonic-bandgap (UC-PBG) structure," IEEE Trans. Microwave Theory Tech., vol. 47, no. 11, pp. 2092-2098, Nov. 1999.

[18] M. N. M. Kehn and P.-S. Kildal, "The N-guide: a novel miniaturized hard quasi-TEM waveguide," in Proc. IEEE Antennas Propag. Int. Symp., 2003, pp. 1111-1114.

[19] M. Kim, J. B. Hacker, A. L. Sailer, S. Kim, D. Sievenpiper, and J. A. Higgins, "A rectangular TEM waveguide with photonic crystal walls for excitation of quasioptical amplifiers," in 1999 IEEE MTT-S Int. Microwave Symp. Dig., Anaheim, CA, Jun. 13-19, 1999, pp. 543-546.

[20] J. A. Higgins, M. Kim, and J. B. Hacker, "The application of photonic crystals to quasioptic amplifiers," IEEE Trans. Microwave Theory Tech., vol. 47, no. 11, pp. 2139-2143, Nov. 1999.

[21] R. Marqués, F. Mesa, J. Martel, and F. Medina, "Comparative analysis of edge- and broadside coupled split ring resonators for metamaterial design-theory and experiments," IEEE Trans. Antennas Propag., vol. 51, no. 10 , pp. 2572-2581, Oct. 2003.

[22] S. Hrabar, J. Bartolic, and Z. Sipus, "Waveguide miniaturization using uniaxial negative permeability metamaterial," IEEE Trans. Antennas Propag., vol. 53, no. 1, pp. 110-119, Jan. 2005.

[23] H.-S. Wu and C.-K. C. Tzuang, "Miniaturized synthetic rectangular waveguide," in 2003 IEEE MTT-S Int. Microwave Symp. Dig., Philadelphia, PA, Jun. 8-13, 2003, pp. 1099-1102.

[24] W. J. Chappell, M. P. Little, and L. P. B. Katehi, "High Q two-dimensional defect resonators-measured and simulated," in 2000 IEEE MTT-S Int. Microwave Symp. Dig., 2000, pp. 1437-1440.

[25] M. J. Hill, R. W. Ziolkowski, and J. Papapolymerou, "A high-Q reconfigurable planar EBG cavity resonator," IEEE Microwave Wireless Comp. Lett., vol. 11, no. 6, pp. 255-257, Jun. 2001.

[26] T.-Y. Yun and K. Chang, "Uniplanar one-dimensional photonic-bandgap structures and resonator," IEEE Trans. Microwave Theory Tech., vol. 49, no. 3, pp. 549-553, Mar. 2001.

[27] K.-P. Me, J. Kim, F.-R. Yang, Y. Qian, and T. Itoh, "Leakage suppression in stripline circuits using a 2-D photonic bandgap lattice," in 1999 IEEE MTT-S Int. Microwave Symp. Dig., 1999, pp. 73-76. 
[28] J. Hesselbarth and R. Vahldieck, "Leakage suppression in coplanar waveguide circuits by patterned backside metallization," in 1999 IEEE MTT-S Int. Microwave Symp. Dig., 1999, pp. 871-874.

[29] J. Yeo and R. Mittra, "Bandwidth enhancement of multiband antennas using frequency selective surfaces for ground planes," in Proc. IEEE Antennas Propag. Int. Symp., 2001, pp. 366-369.

[30] Y. Cassivi and K. Wu, "NRD-guide spurious made suppressor using self-contained periodic planar EBG structure," in 2001 Asia-Pacific Microwave Conf. Dig., 2001, pp. 659-662.

[31] Y. Zhang, J. von Hagen, M. Younis, C. Fischer, and W. Wiesbeck, "Planar artificial magnetic conductors and patch antennas," IEEE Trans. Antennas Propag., vol. 51, no. 10, pp. 2704-2712, Oct. 2003.

[32] T. K. Wu, Frequency Selective Surface and Grid Array. New York: Wiley, 1995.

[33] M. N. M. Kehn and P.-S. Kildal, "Analyzing propagation characteristics of hard waveguides using FDTD," in Proc. IEEE Antennas Propag. Int. Symp., 2002, pp. 790-793.

[34] G. Ruvio, P.-S. Kildal, and S. Maci, "Modal propagation in ideal soft and hard waveguides," in Proc. IEEE Antennas Propag. Int. Symp., 2003, pp. 438-441.

[35] A. A. Kishk, "Analysis of hard surfaces of cylindrical structures of arbitrarily shaped cross section using asymptotic boundary conditions," IEEE Trans. Antennas Propag., vol. 51, no. 6, pp. 1150-1156, Jun. 2003.

[36] H. Uchimura, T. Takenoshita, and M. Fujii, "Development of a laminated waveguide," IEEE Trans. Microwave Theory Tech., vol. 46, no. 12, pp. 2438-2443, Dec. 1998.

[37] H.-S. Wu and C.-K. C. Tzuang, "Miniaturized high-gain synthetic rectangular waveguide antenna of near-omnidirectional radiation pattern," in Proc. Eur. Microwave Conf. EuMC36 Aperture Antennas, Amsterdam, The Netherlands, Oct. 11-15, 2004, pp. 1189-1192.

[38] Ansoft Corporation, Getting Started: An Eigenmode Problem: Ansoft, Jan. 2001, pp. 5-6.

[39] S. Ramo, J. R. Whinnery, and T. Van Duzer, Fields and Waves in Communication Electronics, 3rd ed. New York: Wiley, 1994, pp. 417-428.

[40] C.-K. C. Tzuang, K.-C. Chen, C.-J. Lee, C. C. Ho, and H.-S. Wu, "H-plane mode conversion and application in printed microwave integrated circuit," in Proc. Eur. Microwave Conf., Oct. 2000, pp. 37-40.

[41] C.-J. Lee, H.-S. Wu, and C.-K. C. Tzuang, "A broadband microstrip-towaveguide transition using planar technique," in 2001 Asia-Pacific Microwave Conf. Dig., 2001, pp. 543-546.

[42] N. Jain, N. Kinayman, and E. Channabasappa, "Integrated HMIC planar rectangular-waveguide," in Proc. 2003 Progress Electromagnetics Research Symp., Singapore, Jan. 2003, p. 34.

[43] H.-C. Chen and C.-K. C. Tzuang, "All-planar dual-mode asymmetric filters at Ka-band," IEEE Microwave Wireless Comp. Lett., vol. 13, no. 3, pp. 111-113, Mar. 2003.

[44] W.-C. Lee, S.-C. Lin, and C.-K. C. Tzuang, "Planar realization of low phase noise $15 / 30 \mathrm{GHz}$ oscillator/doubter using surface mount transistors," IEEE Microwave Wireless Comp. Lett., vol. 13, no. 1, pp. 10-12, Jan. 2003.

[45] D. M. Pozar, Microwave Engineering, 2nd ed. New York: Wiley, 1998.

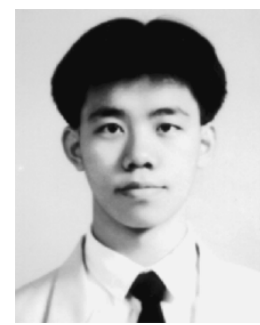

circuits.
Hsien-Shun Wu (S'97) received the B.S. degree in electronic engineering from the National Taipe University of Technology, Taipei, Taiwan, R.O.C., in 1999, and the M.S. degree in communication engineering from National Chiao Tung University, Hsinchu, Taiwan, in 2001, where he is currently working toward the $\mathrm{Ph}$. D. degree in communication engineering.

His research interests include design of wireless system modules and design and development of a photonic bandgap structure for radio-frequency

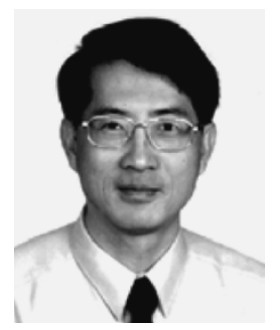

Ching-Kuang C. Tzuang (S'80-M'80-SM'92F'99) received the B.S. degree in electronic engineering from National China Tung University, Hsinchu, Taiwan, R.O.C., in 1977, the M.S. degree from the University of California at Los Angeles in 1980, and the Ph.D. degree in electrical engineering from the University of Texas at Austin in 1986.

From 1981 to 1984, he was with TRW, Redondo Beach, CA, where he was involved with analog and digital microwave monolithic integrated circuits. Since 1986, he has been with the Institute of Communication Engineering, National Chiao Tung University. In February 2004, he joined the Graduate Institute of Communication Engineering, Department of Electrical Engineering, National Taiwan University, Taipei, Taiwan, where he conducts research on advanced guiding structures for research and development of RF system-on-chip, integrating active and passive microwave/millimeter-wave RF signal-processing components into a single chip. His research activities also involve the design and developmen of millimeter-wave and microwave active and passive circuits and the field theory analysis and design of various complex waveguiding structures and large-array antennas. He has supervised 61 M.s. students and $21 \mathrm{Ph} . D$. students. Recently he assisted an interest group in Taiwan promoting standardization and application of millimeter-wave radio-frequency technology and establishing international cooperation.

Dr. Tzuang helped in the formation of the IEEE Microwave Theory and Techniques Society (IEEE MTT-S) Taipei chapter, and served as Secretary, Vice Chairman, and Chairman in 1988, 1989, and 1990, respectively. Recently, he assisted an interest group in Taiwan promoting standardization and application of millimeter-wave RF technology and establishing internationl cooperations. 